\title{
Fracture resistance of endodontically treated premolars restored with direct fiberglass-reinforced composite in MOD cavities.
}

\author{
Fabio Piccioli* \\ Odontological Research Center, São Leopoldo Mandic, 13 - Pte. Preta, Campinas - SP, 13045-755, Brazil
}

\begin{abstract}
Introduction: Direct composite restorations in endodontically treated teeth preserve tooth structure. The objective of this study was to evaluate fracture resistance of premolars with MOD preparation and endodontic treatment restored with composite resin and reinforced with fiberglass tape occlusally or interproximally.

Methods: $\mathbf{N}=\mathbf{3 3}$ sound human upper premolars with similar dimensions were selected. The teeth were randomly divided into 3 groups $(n=11)$, with the control group not receiving any endontontic or restorative preparation. The remaining 2 groups were GO: teeth with endodontic treatment, MOD preparation and cusp coverage with fiberglass tape applied to the occlusal surface and GP: teeth with endodontic treatment, MOD preparation without cusp coverage, but fiberglass tape applied mesially and distally to restore the marginal ridges. All 33 teeth were embedded in acrylic resin and underwent fracture strength test in a universal testing machine $(0.1 \mathrm{~mm} / \mathrm{minute})$ until fracture. The fracture pattern was classified into four types (I, II, III and IV). The fracture resistance values were analyzed using one-way ANOVA $(p<0.05)$ and the fracture patterns using the $G$ test $(p<0.05)$.

Results: The mean fracture values were GC $(690.43 \pm 333.76)$, GO $(857.14 \pm 232.07)$, GP $(850.03 \pm$ 266.36), with no significant difference between the groups. Group GP had the highest frequencies of favorable fractures, followed by GC and then GO $(p=0.004)$.

Conclusion: Adding fiberglass tape did not reduce fracture resistance compared to sound teeth, although fracture patterns were more favorable when fiberglass tape was placed interproximally to restore marginal ridges with composite resin.
\end{abstract}

Keywords: Endodontics, Fiberglass tape, Shear strength, Fracture Pattern.

Accepted on September $26^{\text {th }}, 2019$

\section{Introduction}

Teeth resistance to fracture is reduced as a result of factors such as caries, trauma, cavity preparation, abrasion, erosion, non-carious lesions, age of the patient $[1,2]$ and access cavity for endodontic treatment, which can lead to partial or total loss of the cusps, including root fractures in the posterior teeth in the most severe cases [2-5]. Loss of marginal ridge integrity is the main contributor to reducing fracture resistance of endodontically treated teeth [6]. Cavity preparations for direct restorations may involve the marginal ridges and the cavity design that most adversely affects the remaining tooth structure is Class II MOD, with marked decrease in fracture resistance when compared to sound teeth $[7,8]$. Therefore, preservation of sound tooth structure by selecting direct adhesive restorative approaches can be a fast, low-cost alternative with favorable biomechanical behavior for the rehabilitation of endodontically treated posterior teeth [9]. Fiberglass tapes feature good mechanical properties and can be adapted to specific needs, allowing for preservation of tooth structure, using minimally invasive preparations associated with adhesive techniques [9]. Teeth restored with occlusal fiber have shown significantly higher fracture strength, which has been associated with more favorable fracture patterns than teeth restored without fiberglass reinforcement $[10,11]$. Using fiberglass tape occlusally makes teeth even more resilient than sound teeth [12]. Based on the importance of conservative approaches for long-term preservation of tooth substrate, the aim of this study was to evaluate the fracture resistance and fracture pattern of MOD-prepared teeth with endodontic treatment restored with composite resin combined with fiberglass tape placed either on the occlusal or proximal surfaces.

\section{Material and Methods}

This study was approved by the local research ethics committee, registration CAAE: 37101014.4.0000.5374.

\section{Experimental design}

This was an analytical experimental unifactorial study. The study factor was coronal restoration on 3 levels: sound 
teeth, teeth restored with fiberglass tape interproximally and teeth restored with fiberglass tape occlusally. The response variables were fracture resistance (quantitative) and fracture pattern (semiquantitative).

\section{Teeth selection}

Thirty-three sound human upper premolars were selected following evaluation of visual and radiographic features to ascertain homogeneity of dimensions across the sample. The teeth were cleaned and stored in distilled water at $4{ }^{\circ} \mathrm{C}$ until use for a maximum of 7 days. Eleven teeth were randomly picked for the control group (GC group) and the remaining 22 teeth were prepared for a direct MOD restoration by the same operator. Subsequently, root canal access, endodontic treatment and obturation were also performed by the same operator. Once prepared, the 22 teeth were randomly selected to form two further groups of teeth that would receive a fiberglass tape on two different surfaces prior to restoration, namely the occlusal surface and the proximal surfaces, the GO and GP groups, respectively.

\section{Specimen preparation}

All 33 teeth were dipped into a polyether impression material (Impregum Soft ${ }^{\mathrm{TM}}-3 \mathrm{M}$ ESPE, St. Paul, MNUSA) to simulate the periodontal ligament. The specimens were then embedded in acrylic resin in 3/4 PVC cylinders measuring $2 \mathrm{~cm}$ in height (D). The PVC cylinders containing the specimens were kept in $100 \%$ relative humidity at $37^{\circ} \mathrm{C}$ until the restorations were performed for each experimental group. The teeth from the GO group had a cusp reduction of approximately $2 \mathrm{~mm}$ to allow for the composite resin and the fiberglass tape (Interlig ${ }^{\circledR}$, Angelus, Londrina, PR, Brazil). In the GP, the teeth had a surplus preparation (slicetype preparation) made in the two proximal boxes against the buccal and palatal walls to make room for the Interlig ${ }^{\circledR}$ fiberglass tape, which was then covered with composite resin, in an effort to reinforce the marginal ridges of the two proximal boxes. Prior to restoration, prophylaxis of the coronal enamel was performed with rubber cups and pumice, followed by copious rinsing with distilled water. Acid etching followed using $37 \%$ phosphoric acid gel for thirty seconds on the enamel and fifteen seconds on the dentin, followed by rinsing with water spray for one minute. The cavity was dried with cotton pellets. Two consecutive layers of the 3M ESPM (St. Paul - MN - USA) SingleBond 2 ${ }^{\mathrm{TM}}$ adhesive system were applied to the etched areas using a Microbrush ${ }^{\circledR}$ (Grafton, WI - USA) to ensure a thin and homogeneous layer. Gentle jets of air were used to encourage solvent evaporation and lightcuring followed for 40 seconds using an Optilux Demetron light curing equipment (Kerr, Orange, CA, USA), set for $520 \mathrm{~mW} / \mathrm{cm}^{2}( \pm 20)$ with the aid of an analogic Demetron radiometer (Kerr, Orange, CA, USA).

The final restoration for the GO group was carried out using Filtek Z250 ${ }^{\mathrm{TM}}$ composite resin (3M ESPM, St. Paul MN - USA) in small increments, first against the palatal wall at an angle with the cervical wall, then likewise the buccal wall and so forth until forming a buccal-palatal intercusp at the height of the removed cusp. Each increment was lightcured for 40 seconds. The original Interlig ${ }^{\circledR}$ fiberglass tape from the Angelus factory (Londrina, PR, Brazil) is supplied in 8.5 - length $\times 0.2 \mathrm{~mm}$ width $\times 0.2 \mathrm{~mm}$ thick, which was then trimmed to eight millimeters with scissors. The $8-\mathrm{mm}$ piece of fiberglass tape was adapted onto the cusps in a buccalpalatal direction aided by a pair of clinical forceps and a small Styrofoam ball to accommodate the tape into place. The tape was then light-cured for 40 seconds, as recommended by the manufacturer. The restoration was completed by rebuilding the cusps, ensuring a two-millimeter coverage including fiberglass and resin. The final finish was achieved using three decreasing grains of Sof-Lex ${ }^{\mathrm{TM}}$ discs (3M St. Paul - MN USA), in the sequence recommended by the manufacturer: black (thick type), dark blue (medium type) and medium blue (thin type) all for 15 to 20 seconds with rinsing and drying between discs. The final polishing was performed using the Shofu ${ }^{\text {TM }}$ polishing kit (Higashiyama-Ku-Kyoto-Japan).

The teeth in the GP group were restored with the same aforementioned materials using the incremental technique. The proximal boxes were filled allowing for the $2 \mathrm{~mm}$ needed to accommodate the fiberglass and the composite resin to restore the marginal ridges both mesially and distally in the buccal-palatal direction, finishing the restoration in composite. No cusp reduction was needed for this group.

The excess fiberglass tape buccally and palatally was removed using tapered burs for resin finishing (2200F Microdont ${ }^{\circledR}$, São Paulo, SP, Brazil). The final finish was achieved with Sof-Lex ${ }^{\mathrm{TM}}$, as described for the GO group. The restoration was then polished with the Shofu ${ }^{\mathrm{TM}}$ polishing kit (Higashiyama-Ku- Kyoto-Japan).

\section{Fracture resistance test}

All specimens were submitted to the fracture strength test in a universal testing machine (EMIC ${ }^{\circledR}$ DL -2000 , São José dos Pinhais, PR, Brazil) at $0.1 \mathrm{~mm} /$ minute. Loading was measured in Newton $(\mathrm{N})$ and the specimens were loaded until fracture occurred. The results, the strain and displacement curve were obtained using the EMIC software.

\section{Fracture pattern analysis}

The fractured specimens were radiographed in the buccal-palatal and mesiodistal directions to evaluate possible root fractures and analyzed under a magnifying glass at $3 \mathrm{X}$ magnification to determine the failure pattern, using the classification scale proposed by Burke, as follows: Type I: isolated fracture of the restoration. Type II: Restoration fracture involving small portion of the tooth. Type III: fracture of the restoration involving more than half of the tooth, without periodontal involvement. Type IV: fracture of the restoration involving more than half of the tooth, with periodontal involvement. In other words, this is a 4-point scale to classify tooth fractures from most favorable to least favorable in terms of restorability. 


\section{Statistical analyses}

Normality and equality of variance were ascertained using the Shapiro-Wilk and Levene tests, respectively. Both assumptions were met and the fracture strength data underwent one-way analysis of variance (ANOVA). The fracture patterns were compared using the G-test. Statistical approaches were conducted using a 5\% significance level on SPSS 20 (SPSS Inc., Chicago, IL, USA).

\section{Results}

The one-way ANOVA revealed no significant difference in fracture resistance values among the three groups $(p=$ 0.303 ). The teeth restored with composite resin and reinforced with fiberglass tape following endodontic treatment, were as resistant to fracture as the sound teeth. The location of the fiberglass tapes, either on the occlusal or proximal surfaces, did not influence the resistance to fracture.

The $G$ test $(p=0.004)$ revealed a significant difference in fracture pattern between the GO and GP groups. While in the GP group $27.3 \%$ the fractures were confined exclusively to the restoration (type I failure), in the GO group, this fracture pattern affected only $9.1 \%$ of the teeth. In the GO group, the predominant fracture type was III, in which, in addition to the restoration, more than half of the tooth fractured. In the GP group, the fractures were less severe, based on the predominance of type II fractures, in which only a small portion of the tooth was fractured along with the restoration. Regarding type IV fractures, i.e. those in which there is periodontal involvement, no significant difference was observed between the groups GO and GP.

\section{Discussion}

Fracture resistance is reduced by $63 \%$ in teeth prepared for MOD restorations and is even more compromised when associated with endodontic treatment, with a further 5\% reduction in the final resistance [6]. The highest reductions in fracture resistance are associated to loss of marginal ridge integrity $[4,6,13,14]$. The decrease in fracture resistance is directly related to the number of ridges removed, since they are important reinforcement structures [15]. The inclusion of fiberglass tape in the present study to restore the marginal ridges is therefore justified on such classic studies, which established the role played by such structures in maintaining the integrity of teeth facing occlusal loads.

Studies proposing the use of cusp coverage preparations reinforced with fiberglass tape for direct composite resin restorations revealed an increase in fracture resistance compared to sound teeth in endodontically treated maxillary premolars, in addition to displaying a better fracture pattern in favor of restorability of the affected teeth [10-12,16-19], which is corroborated by the findings presented herein (Table 1).

The results of the present study demonstrate that the location of the fiberglass tapes, either on the occlusal (GO) or proximal (GP) surfaces, did not influence the fracture
Table 1. Mean values (standard deviations) of fracture strength from sound $(G C)$ and restored teeth using glass fiber tape on the occlusal $(G O)$ and proximal (GP) surfaces.

\begin{tabular}{|l|c|}
\hline \multicolumn{1}{|c|}{ Group } & Fracture resistance (N) \\
\hline GC: control (sound teeth) & $690,43(333,76)$ A \\
\hline GO: fiberglass tape on the occlusal surface & $857,14(232,07)$ A \\
\hline GP: fiberglass tape on the interproximal surfaces & $850,03(266,36)$ A \\
\hline
\end{tabular}

resistance values, which were both similar to the group of sound unrestored teeth. Despite a lack of statistical significance, the groups GO and GP presented higher mean values of resistance to fracture in relation to the $\mathrm{GC}$ group of healthy teeth. The null hypothesis has to be accepted therefore, thus corroborating the studies by Oskoee et al. [10], Oliveira et al. [11] and Montanari et al. [12], who advocate the use of fiberglass tape onto the occlusal surface to increase fracture resistance.

Regarding the fracture patterns, the studies by Oskoee et al. [10], Oliveira et al. [11] and Montanari et al. [12] limited themselves to classifying the fractures simply as favorable or unfavorable, considering the cement-enamel junction as the limit for restorability. The results showed that teeth restored with fiberglass tape on the occlusal surface tend to show a high percentage of fractures favoring restorability, which is corroborated by the findings from the present study when fiberglass tape was used to rebuild the marginal ridges on the proximal aspects of the teeth. The aforementioned authors did not however detail the percentage of each type of fracture, according to the classification of Burke et al. [20].

The GP group, in which the fiberglass tape was applied to the proximal cavities with an additional slice-type preparation, showed a significant improvement in fracture pattern, when compared to the GO group. The GP group showed an $81.8 \%$ of types I and II fractures, while group GO had only $18.2 \%$. Regarding the more severe types of fractures, namely types III and IV, which imply more complex restoration procedures, the GP group showed a lower percentage of such fractures (18.2\%) when compared to group GO (81.8\%) (Figure 1). The approach used in the latter group has, however, been proposed by the authors of the most recent studies (Oskoee [10], Oliveira [11] and Montanari [12]). The present study was based on 11 teeth per group, which reached a posthoc sample power of $44.71 \%$ (G*Power, University of Düsseldorf) in multicomparison tests, based on the means and standard deviations obtained from the three groups. In order to achieve a sample power of $80 \%$, a minimum of 45 teeth would be necessary in each group, i.e. $\mathrm{N}=135$ teeth, though no studies in the literature were found investigating a sample size of this magnitude. This would not only be difficult to achieve, based on the strict inclusion and exclusion criteria used in this study, but according to the trend observed from the means, it would only prove that the use of the tape, either occlusally or proximally, would increase the fracture resistance when compared to a sound tooth, which was never the aim of this study. 
Citation: Piccioli F. Fracture resistance of endodontically treated premolars restored with direct fiberglass-reinforced composite in MOD cavities. J Clin Dentistry Oral Health. 2019;3(2):1-5.

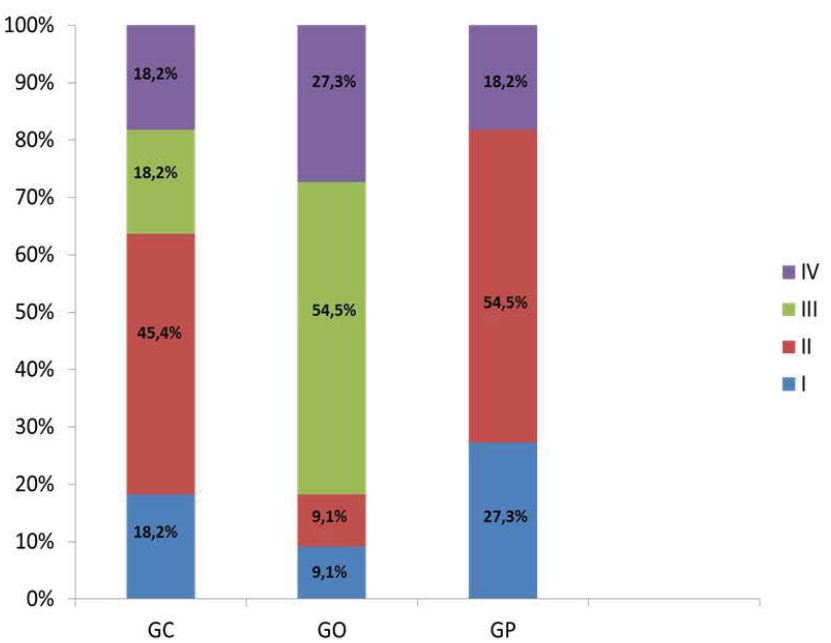

Figure 1. Bar chart of the proportion (\%) of fracture patterns across the experimental groups. The G-test revealed a higher prevalence of less severe fractures in GP compared to GO. Type I: isolated fracture of the restoration. Type II: Restoration fracture involving small portion of the tooth. Type III: fracture of the restoration involving more than half of the tooth, without periodontal involvement. Type IV: fracture of the restoration involving more than half of the tooth, with periodontal involvement.

Increasing the sample size would therefore be a purely numerical exercise with very little practical value. The fact that no statistically significant difference was observed between the groups implies that teeth with fiberglass tape and composite resin may be as resistant to fracture as sound unrestored teeth.

The incorporation of fiberglass tapes into direct composite resin restorations may be an acceptable conservative treatment option for MOD cavities following endodontic treatment in upper premolars, regardless of the surface in which the tape is placed, namely occlusal or proximal. Despite the slight better outcome observed in the latter, the use of fiberglass tapes in proximal surfaces may be hindered by the presence of tightly placed adjacent teeth. This does, nonetheless set the grounds for yet another conservative and relatively low-cost approach to manage weakened teeth.

\section{Conclusion}

Resistance to fracture was not affected by the use of fiberglass tape to reinforce direct composite restorations in MOD cavities compared to sound teeth.

Regarding fracture pattern, the application of fiberglass tape to the proximal surfaces resulted in the most favorable outcomes.

\section{References}

1. Mondelli J, Steagall L, Ishikiriama A, et al. Fracture strength of human teeth with cavity preparations. J Prosthet Dent. 1980;43(4):419-22.

2. Khera JH, Askarieh Z, Jakobsen J. Adaptability of two amalgams to finished cavity walls in Class II cavity preparations. Dent Mater. 1990;6:5-9.
3. Burke FJT. Tooth fracture in vivo and in vitro. J Dent. 1992; 20(3):131-9.

4. Mondelli J. Evolução e Princípios Biomecânicos das Cavidades de Classe II para Amálgama. In: Fundamentos de Dentística Operatória. $4^{\mathrm{a}}$ reimpressão. São Paulo. 2010;p:93-114.

5. KrishanR,PaquéF, OssarehA, etal.Impacts of conservative endodontic cavity on root canal instrumentation efficacy and resistance to fracture assessed in incisors, premolars, and molars. J Endod. 2014; 40:1160-6.

6. Reeh ES, Messer HH, Douglas WH. Reduction in tooth stiffness as a result of endodontic and restorative procedures. J Endod. 1989;15(11):512-16.

7. Pacheco JFM, Conceição EM. Dentes com tratamento endodôntico:como e com o que restaurar? In: Dentística, saúde e estética. Porto Alegre. Artmed. 2002;p297-310.

8. Awawdeh LA, Al-Qudah AA. Root form and canal morphology of mandibular premolars in a Jordanian population. Int Endod J. 2008;41:240-8.

9. Soares PV, Santos-Filho PCF, Queiroz EC, et al. Fracture resistance and stress distribution in endodontically treated maxillary premolars restored with composite resin. J Prosthodont. 2008; 17(2):114-9.

10. Oskoee PA, Ajami AA, Navimipour EJ, et al. The effect of three composite fiber insertion techniques on fracture resistance of root-filled teeth. J Endod. 2009;35(3):413-6.

11. Oliveira CA. Avaliação da influência do tipo de proteção de cúspide em dentes com preparos MOD e tratados endodonticamente na resistência à fratura [TCC]. Campinas: Faculdade de Odontologia da São Leopoldo Mandic de Campinas. 2010.

12. Montanari R. Influência do tipo de restauração na resistência à fratura em dentes tratados endodonticamente [Mestrado]. Campinas: Faculdade de Odontologia da São Leopoldo Mandic de Campinas. 2016.

13. Beltrão MCG. Influência da transfixação horizontal por um pino de fibra de vidro na resistência à fratura de molares endodonticamente tratados [tese]. Rio Grande do Sul: Pontifícia Universidade Católica do Rio Grande do Sul. 2005.

14. Pradeep PR, Kumar VC, Bantwal SR, et al. Fracture strength of endodontically treated premolars: An In-vitro evaluation. J Int Oral Health. 2013;5(6):9-17.

15. Bezerra RB, Nararro MFL, Souza Jr MHS, et al. Influência das cristas marginais na resistência à fratura de dentes despolpados submetidos ao tratamento clareador. Rev. Odontológica Universidade de São Paulo. 1993;7(2):125-9.

16. Oskoee PA, Chaharom ME, Kimyai S, et al. Effect of two types of composite fibers on fracture resistance of 
endodontically treated maxillary premolars: an in vitro study. J Contemp Dent Pract. 2011;12(1):30-4.

17. Luthria A, Srirekha A, Hegde J, et al. The reinforcement effect of polyethylene fibre and composite impregnated glass fibre on fracture resistance of endodontically treated teeth: An in vitro study. J Conserv Dent. 2012;15(4):372-6.

18. Khan SI, Anupama R, Deepalakshmi M, et al. Effect of two different types of fibers on the fracture resistance of endodontically treated molars restored with composite resin. J Adhes Dent. 2013;15(2):167-71.

19. Torabzadeh H, Ghassemi A, Sanei M, et al. The influence of Composite thickness with or without fibers on fracture resistance of direct restorations in endodontically treated teeth. Iran Endod J. 2014;9(3):215-9.

20. Burke FJ, Watts DC. Fracture resistance of teeth restored with dentinbonded crowns. Quintessence Int. 1994;25:335-40.

\section{*Correspondence to:}

Fabio Piccioli

Odontological Research Center

São Leopoldo Mandic

13 - Pte. Preta,

Brazil

Tel: (11)96365-0276

E-mail: fabio.piccioli22@hotmail.com 